Economía: Teoría y PrÁctica • Nueva Época, número 45, julio-diciembre 2016,

pp. 67-96, http://www.izt.uam.mx/economiatyp/ojs

\title{
Capacidad redistributiva de los gastos fiscales en el contexto de la polarización del ingreso en México, 2008-2012*
}

\section{Redistributive Capacity of Fiscal Policy in the Context of Income Polarization in Mexico, 2008-2012}

\author{
José Luis Manzanares Rivera**
}

\begin{abstract}
RESUMEN
El presente trabajo busca abordar el concepto de gasto fiscal a partir del caso del impuesto negativo por enajenación de gasolinas y diesel en México para destacar su potencial redistributivo y analizar el comportamiento territorial de los niveles de polarización económica en el marco de la asignación presupuestal de aportaciones federales de corte social. Se aplican métodos de estimación no paramétrica a los microdatos de la Encuesta Nacional de Ingresos y Gastos de los Hogares, para el periodo 2008-2012. Los resultados sugieren que el gasto fiscal implícito en la tasa negativa del Impuesto Especial sobre Producción y Servicios a la gasolina y el diesel tiene un potencial redistributivo equivalente a una reducción del índice de polarización de Duclos, Esteban y Ray, de IDER $\approx 0.26$ a IDER $\approx 0.23$. Por otra parte, se detecta un patrón de extrema inequidad desde Michoacán hasta Veracruz y Tamaulipas, conformando un cinturón de polarización.
\end{abstract}

Palabras clave: gastos fiscales, inequidad, aportaciones federales, Ramo 33, presupuesto. Clasificación JEL: C15, H23, H31, H53.

\begin{abstract}
In this paper, the relationship between fiscal expenditures and income polarization is documented. Additionally, the spatial behavior of income polarization is examined in the context of selected federal expenditures outlined in the Budget for the period 2008-2012. The analysis is based on micro data from the Encuesta Nacional de Ingresos y Gastos de los Hogares (National Survey of Income and Expenses of Households) following the Duclos, Esteban and Ray methodology to estimate polarization using a non-parametric approach. Results suggest that the fiscal expenditure implicit in the Impuesto Especial sobre Producción y Servicios (Special Tax on Production and Services) has potential to reduce IDER from 0.26 to 0.23 . Also an extreme inequality spatial pattern is identified, which extends from Michoacán to Veracruz and Tamaulipas, forming a polarization belt.
\end{abstract}

Keywords: fiscal expenditures, inequality, federal appropriations, Ramo 33, budget. JEL classification: C15, H23, H31, H53.

\footnotetext{
* Fecha de recepción: 20/03/2014. Fecha de aprobación: 29/02/2016.

** El Colegio de la Frontera Norte. Correo electrónico: jlmanzanares@colef.mx.
} 


\section{INTRODUCCIÓN}

Los esfuerzos de la última década para reducir la pobreza mediante la asignación de recursos vía programas públicos de transferencias, así como la reciente reforma hacendaria en México, dan cuenta de la importancia de un enfoque de gasto público aparentemente orientado a lograr la distribución equitativa del ingreso, sin embargo, tal como se reconoce desde la perspectiva teórica, los efectos del crecimiento son menos efectivos en una sociedad polarizada económicamente. Así, en países emergentes de América Latina caracterizados por altos niveles de desigualdad económica, como Brasil, Colombia o México, se ha documentado recientemente un aumento constante de la polarización del ingreso (Huesca, 2003; Carrillo-Huerta y Vázquez, 2005; Gasparini et al., 2009; Fuentes y Fuentes, 2010; Clementi y Schettino, 2013), lo que, entre otros efectos sociales, podría aminorar los beneficios del crecimiento económico y, en particular, el impacto de los recursos asignados mediante políticas redistributivas en favor de los pobres (pro-poor), hacia las que aparentemente transitan algunas naciones de la región.

En México, un país caracterizado por un bajo crecimiento económico ${ }^{1} \mathrm{y}$ una débil capacidad fiscal, el análisis de la polarización económica con una perspectiva territorial, en el contexto de la existencia de tratamientos fiscales diferenciados como los contenidos en el Presupuesto de Gastos Fiscales, adquiere pertinencia por su potencial redistributivo. Formalmente, el balance entre las acciones de política fiscal -mediante la estructura de gasto público- y el bienestar ha sido abordado en la literatura sobre la distribución del ingreso y sobre la pobreza, lo que ha permitido tener una base teórica cada vez más robusta para realizar acercamientos empíricos. Algunos autores como Sen (1973, p. 92), Kakwani (1976), Atkinson (1997), y Bourguignon y Morrison (2002) han aportado elementos particulares que contribuyen a éste planteamiento conceptual.

Los aspectos redistributivos de las medidas fiscales en materia de combustibles se han abordado a menudo desde la perspectiva teórica de la presencia de externalidades, ya que se asume que en este caso los sistemas del fisco tienen la capacidad de internalizar los impactos negativos que el consumo de combustibles como la gasolina supone. Esfuerzos recientes en esta perspectiva incluyen la serie titulada Companion to the Inventory of Support Measures for Fossil

\footnotetext{
${ }^{1}$ Según cifras del Banco Mundial (The World Bank, s.f.), el crecimiento anual promedio del producto interno bruto (PIB) de México en el periodo 2000-2012 fue de 2.4\%, el menor entre las principales economías de América Latina, incluyendo Brasil, Argentina, Chile, Colombia y Venezuela.
} 
Fuels, sobre el apoyo presupuestal y los gastos fiscales por combustibles en países miembros de la Organización para la Cooperación y el Desarrollo Económicos (OCDE) (OECD, 2015). En el ámbito latinoamericano, Agostini y Jiménez (2015) enfatizan su potencial como instrumento de política en la discusión sobre el grado de regresividad de los impuestos a la gasolina.

Por su parte, Antón-Sarabia y Hernández-Trillo (2014) argumentan la importancia de estimar el nivel impositivo óptimo sobre la gasolina en el caso de México, un país de menor desarrollo respecto a Estados Unidos (EU), y aplicando la metodología de Parry y Small (2005), que permite identificar los componentes del impuesto óptimo, demuestran que la existencia de subsidios (gravámenes negativos como el Impuesto Especial sobre Producción y Servicios) $)^{2}$ explica aproximadamente $20 \%$ de la diferencia entre el impuesto óptimo a la gasolina en México y el reportado en Eu por Parry y Small (2005). Aquellos autores sugieren el potencial redistributivo del nivel impositivo óptimo al indagar el efecto de éste por deciles y concluyen que, de hecho, su efecto es progresivo, dado que sólo 9\% de los hogares de menores ingresos, o pobres, poseen automóvil, mientras que $85 \%$ de las familias de mayores ingresos, o afluentes, sí requieren gasolina, ya que $91 \%$ de ellas posee por lo menos un automóvil (Antón-Sarabia y Hernández-Trillo, 2014, p. 565).

Sin embargo, aunque la afirmación de dichos investigadores en el sentido de que la progresividad del impuesto óptimo es acertada en términos absolutos, no es así en términos relativos. La lógica es simple: en términos relativos, el impuesto a la gasolina representa una mayor proporción del ingreso de los hogares de los deciles con menores ingresos que la correspondiente a las familias afluentes, a pesar de que los primeros son menos que las segundas. Lo anterior, no obstante, permite notar que los instrumentos fiscales aplicados a los combustibles y, particularmente, a la gasolina representan un área de creciente interés en los países de América Latina.

Adicionalmente, si se consideran los aspectos redistributivos y pesar de que la formalización teórica de la medición de la polarización del ingreso se ha consolidado en las últimas décadas, en México se tienen escasas aplicaciones empíricas de los algoritmos para medir esta polarización. Notablemente, el trabajo pionero de Huesca (2003) pone de relieve el tema en el contexto del análisis de la clase media, implementando los algoritmos desarrollados por Esteban y Ray (1994) y Esteban, Gradín y Ray (1999) en un entorno de estimación para-

\footnotetext{
${ }^{2}$ En un escenario en el que el precio de importación supera el precio interno.
} 
métrica con datos agregados a escala nacional para mostrar el tránsito del país hacia una sociedad en la que al parecer la clase media ha tendido a desaparecer.

Desde una perspectiva teórica, sin embargo, la aplicación de la propuesta de Esteban y Ray (1994) implica al menos una desventaja metodológica, tal como puntualizan Duclos, Esteban y Ray (2004, p. 1739); ésta consiste en que si bien el espacio del análisis de la distribución del ingreso sobre el cual está calculado el índice del mencionado trabajo es continuo, la elección de los grupos de ingreso considera un conjunto finito discreto, preestablecido de forma exógena, lo que implica la existencia de inconvenientes discontinuidades entre los grupos de ingreso (Esteban y Ray, 1994, p. 846).

Además, se reconoce que los niveles de polarización en la sociedad difieren tanto geográfica como temporalmente. En principio, entender estas variaciones territoriales y cronológicas es importante para la evaluación de medidas de política pública, por lo que un análisis que considere la desagregación a nivel estatal podría contribuir a comprender la dinámica del fenómeno en México.

El objetivo del presente trabajo es abordar el concepto de gasto fiscal a partir del caso del impuesto negativo a la enajenación de gasolinas y diesel en México y examinar la capacidad redistributiva de este esquema fiscal. La exposición se organiza de la siguiente manera: en la primera sección se presenta el gasto fiscal asociado al Impuesto Especial sobre Producción y Servicios (IEPS); en la siguiente, se determina el nivel de inequidad en las entidades federativas mediante el cálculo del índice de polarización económica y se establece el efecto de este gasto fiscal en el escenario de su reasignación a programas focalizados en el gasto social reconocidos por su carácter progresivo. Por último, se discuten los resultados y se presentan las conclusiones.

\section{IMPUESTO NEGATIVO A LA ENAJENACIÓN DE GASOLINAS Y DIESEL: CONCEPTO, MEDICIÓN Y POTENCIAL REDISTRIBUTIVO}

En esta sección se presenta el concepto y evolución de la tasa negativa del IEPS a la enajenación de gasolinas y diesel, se muestra su carácter regresivo y se contrasta con usos alternativos para destacar su potencial redistributivo en el caso de México durante el periodo 2008-2012. En este lapso las condiciones internas, así como los factores adversos vinculados a la crisis de la economía mundial que afectaron a México, se reflejaron en un gasto fiscal del IEPs a la enajenación de gasolinas y diesel equivalente a $1.25 \%$ del PIB, en promedio, lo que constituye la magnitud más elevada de la última década. 
Tal como se reconoce en la literatura, uno de los objetivos extrafiscales del sistema tributario es incidir en la distribución del ingreso (Musgrave, 1969, p. 390) y, en este sentido, uno de los instrumentos para lograr tal meta son los gastos fiscales. De acuerdo con la definición original de Surrey (1973), en este trabajo dichos gastos se entienden como apoyos indirectos en forma de disminuciones, exenciones o diferimientos otorgados por el gobierno a contribuyentes específicos, por lo que no representan una erogación explicita. Este concepto es relevante, ya que su estimación permite cuantificar, por una parte, el impacto de tales apoyos en la reducción de los ingresos tributarios y, por otra, permite evaluar su efecto para el logro de objetivos extrafiscales, como la redistribución del ingreso.

No obstante su importancia como mecanismo de estimación monetaria de la pérdida recaudatoria debida a los tratamientos fiscales diferenciales, el registro formal de gastos fiscales en México es relativamente reciente. Si bien tiene su antecedente en el Registro Único de Transferencias (RUT), presentado por primera vez en 1983 (Mijangos, 1991, p. 478), ${ }^{3}$ es apenas a partir de 2002 que se ha tenido un registro formal y sistemático mediante un presupuesto, lo que contrasta con el entorno internacional, en el que esta práctica es común desde los ochenta (Ley de Ingresos de la Federación para el Ejercicio Fiscal de 2002, 2002, cap. 4).

De acuerdo con el marco tributario, los gastos fiscales se originan por la existencia de tratamientos diferenciales en la estructura impositiva (IMF, 2007, p. 76). Así, el gasto fiscal derivado del IEPs a la enajenación de gasolinas y diesel, se prevé en la Ley de Ingresos de la Federación para el Ejercicio Fiscal de 2013 (2012, cap. 2, art. 7, frac. II, § 5) en el caso en el que la tasa aplicable a Pemex por el mencionado concepto resulte negativa, lo cual ocurre cuando el precio internacional de referencia, $P^{*}$, es superior al precio de venta al consumidor en el mercado interno, $P^{c}$. Sea $S$ : la tasa negativa de impuesto, entonces $S=P^{*}-P^{c} / P^{*}>P^{c}$. Esta diferencia de precios propicia una pérdida de recaudación por concepto IEPS. ${ }^{4}$ En el caso de la gasolina Magna, el precio internacional de referencia establecido por la Secretaría de Hacienda y Crédito Público (SHCP) es el promedio del precio de contado de la gasolina Unleaded Regular-87, vigente en la costa estadounidense del golfo de México, mientras que el de la gasolina Premium

\footnotetext{
${ }^{3}$ Este instrumento rebasa conceptualmente la noción de gasto fiscal. No obstante, su uso ha permitido integrar el Presupuesto de Gastos Fiscales que se presenta anualmente en México desde 2002.

${ }^{4}$ Conforme se establece en el artículo 7, fracción II de Ley de Ingresos de la Federación para el Ejercicio Fiscal de 2013 (2012), cuando la tasa de este impuesto resulta negativa $\left(P^{*}>P^{c}\right)$, Pemex puede acreditar el monto del IEPS a su favor, contra el IVA o el Derecho Ordinario sobre Hidrocarburos.
} 
Gráfica 1. Evolución del gasto fiscal por concepto de tasa negativa del IEPS a gasolina y diesel en comparación con rubros de gasto seleccionados, 2008-2012 (millones de pesos mexicanos constantes, año base 2010)

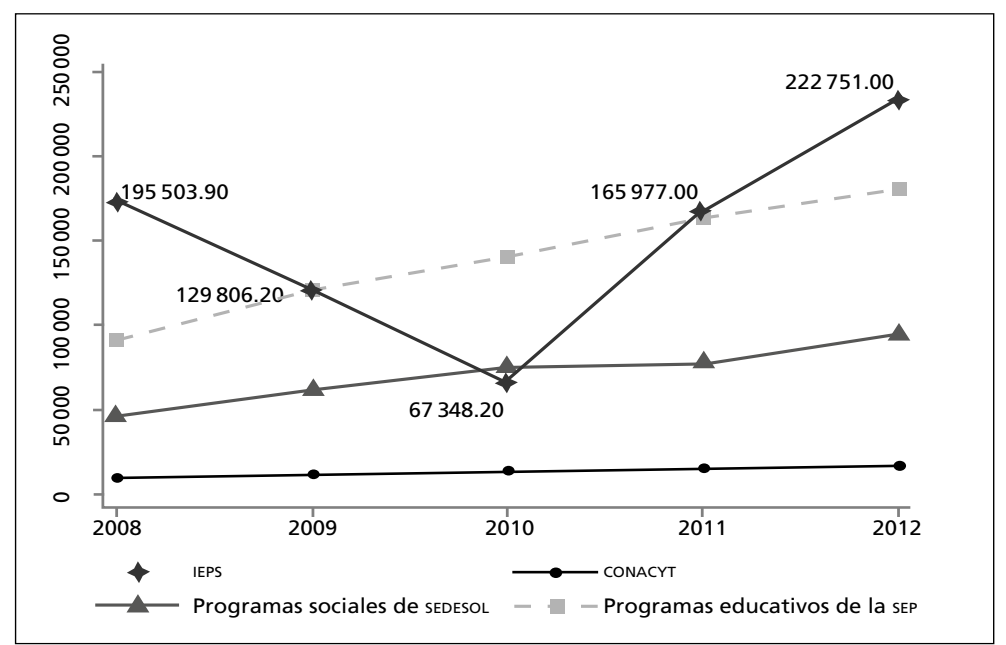

Nota: IEPS = gasto fiscal por tasa negativa del impuesto a la enajenación de gasolina y diesel, consignado en el Presupuesto de Gastos Fiscales (SHCP, 2008; 2009; 2010; 2011; 2012a). CONACYT $=$ recursos para programas consignados en CONEVAL (2014). Programas sociales de SEDESOL = recursos para los tres principales programas de gasto social, por asignación presupuestal: Oportunidades, 70 y Más y Programa para el Desarrollo de Zonas Prioritarias de acuerdo a CONEVAL (2014). Programas educativos de la SEP = presupuesto asignado para programas educativos de la SEP excluyendo subsidios federales para organismos descentralizados estatales, de acuerdo a CONEVAL (2014).

Fuente: Elaboración propia con datos de CONEVAL (2014) y SHCP (2008; 2009; 2010; 2011; 2012a).

corresponde al de la gasolina Unleaded Premium 93 y el del diesel, el del Fuel Oil \#2 Low Sulphur, ambos en la misma zona geográfica.

La definición se acota al gasto fiscal por concepto de IEPS cuando su tasa es negativa por dos razones: primero, debido a su importancia económica, ya que México es un importador neto de este combustible, con una dependencia proyectada promedio, entre 2011 y 2026 , de $42 \%$ de la demanda nacional (Secretaría de Energía, 2012, p. 142). ${ }^{5}$ De hecho, la demanda de gasolina en México es elevada en relación con países de desarrollo similar, con un consumo superior en $198 \%$ al de Argentina, 306\% al de Brasil y $117 \%$ al de Chile (SHCP, 2012b). Segundo, por la persistencia de un diferencial entre el precio de importación y el precio al con-

${ }^{5}$ Según cifras de la Secretaría de Energía (2012), se prevé un crecimiento en la demanda de $71.25 \%$ entre 2011 y 2026. 
Gráfica 2. Fluctuación del tipo de cambio y del precio de referencia del petróleo, 2008-2012

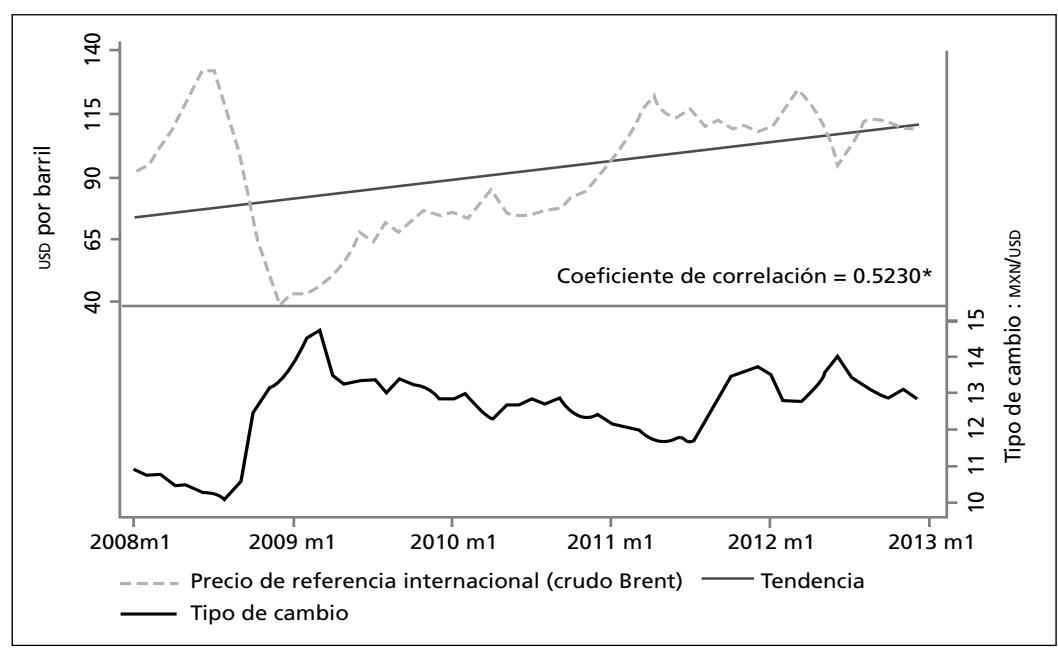

* Estadísticamente significativo a 0.05 .

Nota: El precio al contado del crudo Brent se toma como referente internacional para estimar la variación en el precio de este energético. Serie de periodicidad mensual; $\mathrm{m} 1=$ =inicio del año.

Fuente: Elaboración propia con datos del Banxico (s.f.) y la us Energy Information Administration (s.f.).

sumidor, lo que da lugar a la aplicación de una tasa impositiva negativa que se traduce en un gasto fiscal de magnitud considerable.

El monto del impuesto negativo por concepto de IEPS alcanzó, en el periodo 2008-2012, 781386.3 millones de pesos, monto superior al presupuesto asignado a los programas educativos de la Secretaría de Educación Pública (SEP) en el mismo periodo (CONEVAL, 2014) ${ }^{6}$ y también mayor al presupuesto ejercido en conjunto por los principales programas de gasto social: Oportunidades, 70 y Más y el Programa para el Desarrollo de Zonas Prioritarias. La gráfica 1 registra la magnitud de este recurso en comparación con rubros de gasto seleccionados. Si bien la magnitud del gasto fiscal asociado a la tasa negativa de IEPS es considerable respecto al gasto en otras áreas prioritarias para el país, es evidente también su volatilidad en el periodo, lo que se vincula a fluctuaciones en dos variables externas: el tipo de cambio y el precio del petróleo. La gráfica 2 muestra las variaciones de ambos determinantes.

\footnotetext{
${ }^{6}$ Excluyendo los subsidios federales para organismos descentralizados estatales, de acuerdo con el CONEVAL (2014).
} 
Estos factores determinantes de la tasa negativa del IEPS a la enajenación de gasolina y diesel permiten inferir la influencia exógena en la generación de este gasto fiscal. Durante el periodo que se analiza, el comportamiento del tipo de cambio permitió compensar parcialmente la presión del incremento del precio de referencia del petróleo, lo que resulta evidente durante el primer semestre de 2008, cuando se observa un crecimiento importante en el precio por barril de Brent acompañado de un periodo de apreciación del peso mexicano frente al dólar.

Asimismo, este efecto compensatorio resulta de consideración a partir del primer semestre de 2009, cuando se tiene un lapso de apreciación del tipo de cambio que se extiende hasta el segundo semestre de 2011, en conjunto con un continuo incremento del precio del Brent, lo que contribuye a disminuir la magnitud del gasto fiscal asociado al impuesto negativo. Este efecto es corroborado por las series, que muestran una correlación inversa significativa estadísticamente con un coeficiente de 0.523

Gráfica 3. Consumo de gasolina Magna por estrato socioeconómico, 2012.

Curva de Lorenz y curvas de densidad

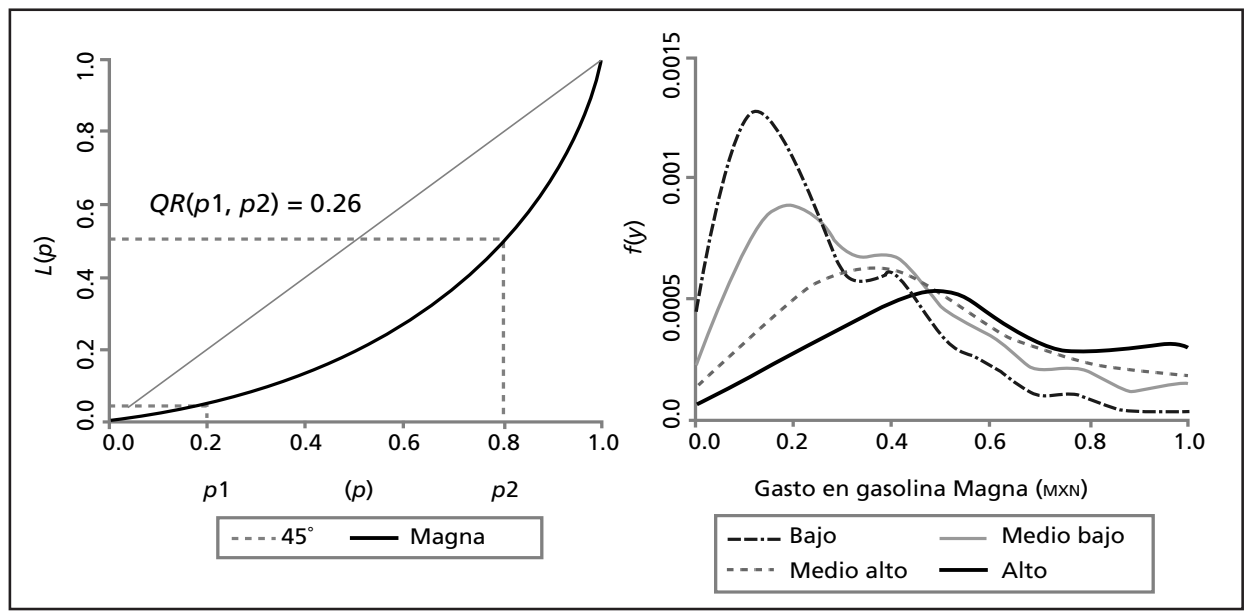

Nota: El gasto se refiere al monto mensual declarado para el mes de referencia; los montos fueron deflactados usando como referencia el Índice Nacional de Precios al Consumidor de agosto 2010. El nivel socioeconómico se refiere a las categorías establecidas por el Instituto Nacional de Estadística y Geografía (INEGI) y se estandarizaron para usar el tamaño del hogar como variable de control.

Fuente: Elaboración propia con datos de la ENIGH (INEGI, 2012b), nueva construcción. 
Otro elemento que ha generado controversia sobre este impuesto negativo es su carácter de apoyo indirecto generalizado, ya que impacta a todos los consumidores de gasolina y diesel y, dada la distribución del ingreso en México, puede implicar un problema de inequidad vertical en el sentido descrito por Musgrave (1990, p. 113), Kraan (2004, pp. 133-134) y Ravallion, Thorbecke y Pritchett (2004, p. 12) Se analiza enseguida la distribución del gasto de los hogares en estos combustibles con el objeto de mostrar su potencial redistributivo. En primer lugar, se estima la tasa de participación del gasto entre el percentil $0.20 \mathrm{y}$ el percentil 0.80 de la distribución del gasto familiar en gasolina de tipo Magna, ${ }^{7}$ con datos oficiales de la Encuesta Nacional de Ingresos y Gastos de los Hogares (ENIGH), instrumento que es un referente estándar en la literatura sobre distribución del ingreso en México, en su edición de 2012 (INEGI, 2012b). El cálculo se acompaña de una curva de Lorenz que da cuenta de que el gasto del $20 \%$ de los hogares de menor consumo sólo representa el 26\% del gasto que realiza el $20 \%$ de los hogares de mayor consumo (gráfica 3).

La parte derecha de la gráfica muestra las curvas de densidad estimadas para la distribución del gasto en gasolina Magna con la finalidad de precisar el carácter regresivo en términos absolutos de la tasa negativa del IEPS al contrastar el gasto por estrato socioeconómico. Se constata que la población del estrato bajo concentra su consumo en la parte inferior de la distribución, en un rango de entre MXN 300 y MXN 400 mensuales, sin embargo a medida que el nivel socioeconómico aumenta, el consumo se desplaza hacia la valores superiores de la distribución. Así, los hogares de estrato socioeconómico alto tienen un gasto mensual que oscila entre los MXN 1200 y los MXN 2000 mensuales en gasolina Magna, lo que implica que es el que recibe en mayor medida la transferencia indirecta de recursos implícita en el gasto fiscal por la tasa negativa del IEPS.

Si bien el consumo de la Magna es representativo de las preferencias de la población, la existencia de otro tipo de gasolina, con un precio mayor, también tiene influencia en el comportamiento del consumo y, en consecuencia, en el patrón de regresividad de la tasa negativa del impuesto. Para verificar este comportamiento, se realizó el contraste entre las curvas de Lorenz correspondientes a la distribución del gasto en gasolina Magna y en gasolina Premium.

\footnotetext{
${ }^{7}$ Se eligió este tipo de gasolina para la estimación porque es la de consumo más usual entre la población, como se infiere de que $92 \%$ de los registros de gasto de gasolina recogidos por la ENIGH 2012 (INEGI, 2012b). Sólo 8\% de los registros corresponden al tipo Premium, lo que es indicativo de las preferencias de consumo de los hogares.
} 
Es considerada, adicionalmente, su incidencia en el ámbito territorial, ya que se asume que los patrones de consumo no son homogéneos a lo largo del territorio, sino que, por el contrario, varían entre las localidades rurales y las ciudades (intermedias y de gran tamaño) lo que influye en el carácter regresivo de la tasa negativa del impuesto. En el lado derecho de la gráfica 4 se muestran las diferencias entre las respectivas curvas de Lorenz para localidades de tres tamaños distintos: ${ }^{8}$ localidades rurales muy pequeñas, de no más de 2500 habitantes; asentamientos urbanos de tamaño medio, de entre 15000 y 99000 habitantes, y localidades mayores de 100000 habitantes.

Gráfica 4. Diferencias entre curvas de Lorenz para gasto en gasolina Magna vs.

Premium, por tamaño de localidad, 2012

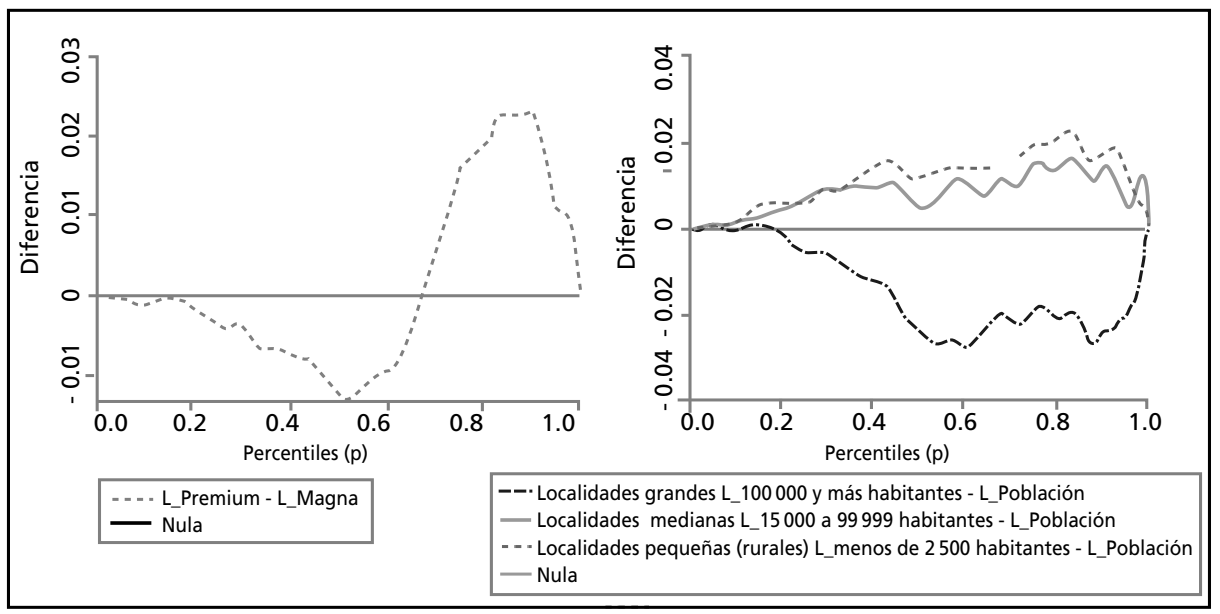

Nota: El gasto se refiere al monto mensual declarado para el mes de referencia. Los montos fueron deflactados usando como referencia el Índice Nacional de Precios al Consumidor de agosto de 2010. Se estandarizó el gasto considerando el tamaño del hogar.

Fuente: Estimación propia con datos de la ENIGH (INEGI, 2012b).

Al comparar Magna con Premium, se observa que en los deciles de menor ingreso (con mayor énfasis del primero al quinto) la distribución del gasto en gasolina Premium exhibe una mayor concentración respecto a la Magna. Este comportamiento de mayor inequidad se traduce en un impacto regresivo más acentuado para este tipo de gasolina respecto a la presentación Magna en térmi-

\footnotetext{
${ }^{8}$ Se siguen los rangos poblacionales establecidos por el INEGI para clasificar las localidades según su tamaño.
} 
nos de la tasa negativa del impuesto, de tal forma que se tiene una relación directamente proporcional entre el grado de desigualdad y el nivel de regresividad, rasgo que que se agudiza con el consumo de gasolina Premium. Es sólo en los deciles superiores ( 8 y 9) que el gasto en gasolina Premium exhibe mayor equidad respecto a la distribución del gasto en Magna, comportamiento que se explica intuitivamente por la relación entre el mayor precio de aquélla y el nivel de ingreso de los hogares en la parte superior de la distribución.

Respecto al contraste por tamaño de localidad, se observa que existe mayor inequidad en el gasto de gasolina en las zonas rurales en comparación con las localidades de mayor tamaño. Así, los hogares de las localidades eminentemente rurales de hasta 2500 habitantes presentan un patrón de mayor concentración del gasto en gasolina en los deciles superiores con respecto a la población total y, por ende, que permite distinguir el carácter de mayor regresividad de la tasa negativa del IEPS en dicho ámbito territorial.

\section{ESTIMACIÓN DEL ÍNDICE DE POLARIZACIÓN ECONÓMICA A ESCALA ESTATAL Y SU RELACIÓN CON LA CAPACIDAD REDISTRIBUTIVA DE LOS GASTOS FISCALES}

En esta sección se analizan los efectos redistributivos del gasto fiscal generado por la tasa negativa del IEPS a gasolina y diesel sobre el grado de polarización económica en México. Dos supuestos sustentan este planteamiento: primero, se asume que el concepto de polarización económica está definido como una expresión extrema de inequidad y, en segundo lugar, que el esquema tributario representa una vía para influir sobre la distribución del ingreso. Así, el enfoque que se sigue pone énfasis en mejorar la distribución del ingreso.

"Es preciso ir más allá de las intervenciones para reducir la pobreza y formular e implementar políticas públicas enfocadas a reducir la desigualdad" (Lopez-Calva y Rocha, 2012, p. 5). Tal como reconoce el citado autor en su análisis sobre el caso de la inequidad en Brasil, y que resulta emblemática para el tema en América Latina, la reducción de la brecha socioeconómica es un requisito para capitalizar los beneficios del crecimiento $\mathrm{y}$, adicionalmente, mejorar el impacto de los recursos asignados mediante políticas públicas a mitigar las condiciones de pobreza.

Por otro lado, como se hizo evidente en la sección anterior, la magnitud del gasto fiscal por la tasa negativa del IEPs ha superado en los últimos años el presupuesto asignado a rubros de gasto social, como el combate a la pobreza o 
los programas educativos, por lo que cabe preguntarse cuál es el efecto, en términos de la polarización económica en México, de la asignación de recursos implícita en la tasa negativa del impuesto a gasolina y diesel sobre los programas sociales que tienen una incidencia progresiva reconocida en la distribución del ingreso.

\section{Datos}

El cálculo del índice de polarización se realizó utilizando la información oficial sobre los ingresos de los hogares en México para el año 2012, la cual se consigna en el Módulo de Condiciones Socioeconómicas, elaborado conjuntamente por el Consejo Nacional de Evaluación de la Política de Desarrollo Social (CONEval) y el Instituto Nacional de Estadística y Geografía (INEGI) (INEGI, 2008; 2012a). Esta base de datos tiene la ventaja, a diferencia de la versión tradicional de la ENIGH, de que permite representatividad a nivel estatal. La muestra contiene 57274 hogares y una vez aplicado el factor de expansión permite estimaciones estadísticamente representativas a nivel nacional con desagregación estatal. La variable que se usa para estimar la polarización es el ingreso corriente por hogar, la cual se normaliza para controlar por tamaño de hogar usando la equivalencia de número de personas por hogar. Las observaciones de la muestra se ponderan con el factor de expansión para considerar las características de muestreo de la encuesta.

La información acerca del presupuesto asignado a los programas sociales se obtuvo del Presupuesto de Egresos de la Federación para el Ejercicio Fiscal 2012 (2012), en el rubro de Aportaciones Federales para Entidades Federativas y Municipios, o Ramo 33, el cual contiene ocho fondos. Si bien los recursos asignados mediante este esquema de trasferencias incide de forma indirecta en los niveles de inequidad de la población, los recursos dirigidos a atender la generación de capacidades educativas tienen un impacto positivo significativo, según se ha documentado mediante el paradigma de capital humano (Litschig y Morrison, 2013), por lo que se incluye como variable en el modelo el Fondo de Aportaciones para la Educación Tecnológica y de Adultos (FAETA).

Así mismo, existe consenso sobre el impacto favorable sobre los recursos destinados a proveer servicios de salud públicos (Organización Mundial de la Salud, 2008), ya que permiten reducir la carga desproporcionada que los sectores de menores ingresos enfrentan, por lo que se incluye el Fondo de Aportaciones para Servicios de Salud (FASSA). También se incorpora el Fondo de Aportaciones para la Infraestructura Social (FAIS), ya que, según lo establece la 
propia Ley de Coordinación Fiscal (2016), en su artículo 33, son inversiones que benefician directamente a la población de las regiones marginadas.

Con esta lógica, y siguiendo un principio de parsimonia en el análisis, se descartan el Fondo de Aportaciones para la Seguridad Pública (FASP) y el Fondo de Aportaciones Múltiples (FAM), dado que sus efectos no son pertinentes para la redistribución del ingreso. Así mismo, se omiten el Fondo de Aportaciones para el Fortalecimiento de las Entidades Federativas (FAFEF) y el Fondo de Aportaciones para el Fortalecimiento Municipal (FORTAMUN), dado que se orientan a reforzar las estructuras administrativas. En particular, el último tiene como objetivo contribuir al saneamiento financiero de las haciendas municipales, lo que rebasa el planteamiento del presente análisis.

Adicionalmente, se consideran los recursos por incentivos a la venta final de gasolinas y diesel que corresponden a las participaciones federales del Ramo 28 del gasto público federal, los cuales se asignan a las entidades federativas en función del consumo efectuado en su territorio y, en menor medida, considerando criterios redistributivos, ya que una fracción de 2/11 se asigna a un fondo de compensación destinado a las diez entidades de menor PIB (Tépach, 2013).

\section{Concepto y estimación del índice de polarización con desagregación estatal}

Es necesaria una precisión preliminar respecto al índice de polarización estimado en este trabajo: la aplicación empírica sigue la estrategia metodológica desarrollada por Duclos, Esteban y Ray (2004), cuya innovación principal es el uso de métodos no paramétricos para el cálculo de dicho índice. Las aplicaciones disponibles hasta el momento, en el caso de México, de los algoritmos para estimar índices de polarización se basan en técnicas de estimación paramétricas, ${ }^{9} \sin$ embargo, como señalan los autores citados: "with sample data aggregated along in-come intervals, it is unclear how to provide a statistically satisfactory account of whether distributive measures (based on such data) are significantly different across time or entities" (Duclos, Esteban y Ray, 2004, pp. 1738). Por ello, plantean una extensión del índice de polarización con base en una estimación no paramétrica: "A rigorous axiomatic development of the polarization concept in the 'density case' is then a prerequisite for proper statistical examination of polarization” (Duclos, Esteban y Ray, 2004, p. 1738).

\footnotetext{
${ }^{9}$ Véase el cuadro A1 del anexo para un listado de los artículos en revistas indexadas sobre el caso de México, con la metodología usada pare estimar la polarización.
} 
En estos autores, la derivación axiomática del índice de polarización parte -al igual que el índice de Esteban y Ray (1994)- de la hipótesis de que el grado de polarización está generado por la interacción de dos fuerzas: identidad con el propio grupo de ingreso y alienación del resto de los individuos en la distribución. Formalmente esta interacción se expresa por $P_{\alpha}(f)=\bar{a}_{\alpha}[1+\rho] .{ }^{10}$

Con este marco, su planteamiento resulta en una forma funcional cuyo parámetro de sensibilidad $\alpha$ (que captura el poder del "efecto de identificación") se ubica en el intervalo [0.25, 1$]$, a diferencia del índice de polarización propuesto por Esteban y Ray (1994), cuyo intervalo es $\alpha$ [1-1.6]. Como demuestran los autores, el coeficiente de Gini resulta en un caso particular del indicador de polarización para el escenario en que el coeficiente que mide el efecto identificación toma el valor $\alpha=0$.

Siguiendo a Duclos, Esteban y Ray (2004), y dado que la innovación en la extensión propuesta por ellos es la aplicación de métodos no paramétricos en un dominio de densidades continuas en $\mathrm{R}_{+}$, se muestra a continuación la derivación para el estimador de polarización en el espacio continuo para una muestra aleatoria $n$ con observaciones independientes e idénticamente distribuidas de ingreso $y_{i}, i=1, \ldots n$ ordenado tal que $y_{1} \leq y_{2} \leq \ldots \leq y_{n}$ para una función de distribución $F$ con densidad asociada $f$ de media $\mu$ que está dando la función

$$
P_{a}(F)=\int_{y} f(y)^{\alpha} a(y) d F(y)
$$

para $a(y) \equiv \mu+y(2 F(y)-1)-2 \int_{-\infty}^{y} x d F(x)$.

Un estimador de (1) es:

$$
P_{a}(F)=n^{-1} \sum_{1=1}^{n} \hat{f}\left(y_{i}\right)^{\alpha} \hat{a}\left(y_{i}\right)
$$

${ }^{10}$ Siguiendo la derivación realizada por Duclos, Esteban y Ray (2004), el autor parte de la definición de las nociones de identificación y alienación entre individuos con ingreso que pertenece al soporte de la función de densidad $f$. Presenta la función de antagonismo efectivo total como $T(i, a)$, donde $i=f(x)$, lo que implica que el individuo de ingreso $x$ siente un grado de identidad que depende de la densidad en $x, f(X)$ y $a=|x-y|$ un grado de alienación cuya distancia es monotónica. En este contexto, la noción de polarización se define como la suma de los antagonismos efectivos $P(F)=\iint T(f(x), x-y) f(x) f(y) d x d y$. Respecto a la interacción $P_{\alpha}(f)=\bar{a} \bar{i}_{\alpha}[1+\rho]$., se tiene que $\bar{a}$ indica el grado de alienación promedio entre dos individuos $x$ y $y$ a partir de la función $\bar{a}=\int a(y) d F(y)=\int|x-y| d F(x) d F(y)$ con $a(y)=\int|y-x| d F(x) ; \bar{i}_{\alpha}$ representa el grado de identificación promedio $\bar{i}_{\alpha} \equiv \int f(y)^{\alpha} d F(y)=\int f(y)^{1+\alpha} d y$. y $\rho$ define la covarianza normalizada entre identificación y alienación $\rho \equiv \frac{\operatorname{cov}_{i_{\alpha}} a}{\overline{i_{\alpha}} \bar{a}}$. 
El estimador $\hat{f}\left(y_{i}\right)^{\alpha}$ a se obtiene aplicando métodos no paramétricos Kernel. Este procedimiento usa un estimador Kernel simétrico $k(u)$ tal que $\int_{-\infty}^{\infty} K(U) d U=1$. Y el estimador $\hat{f}(y)$ es definido como:

$$
\begin{gathered}
\hat{f}(y) \equiv n^{-1} \sum_{1=1}^{n} K_{h}\left(y-y_{i}\right) \\
K_{h}(Z) \equiv h^{-1} K(z / h)
\end{gathered}
$$

con $h$, que es un parámetro de ancho de banda óptimo $h^{*}$.

De acuerdo con el desarrollo llevado a cabo por Duclos, Esteban y Ray (2004, p. 1750), dado un tamaño muestral n, para seleccionar el ancho de banda óptimo comúnmente se minimiza el error estándar medio del estimador a partir de la regla:

$$
h^{*} \approx 4.7 n^{-0.5} \sigma \alpha^{0.1}
$$

El trabajo de Duclos, Esteban y Ray (2004) muestra a profundidad el algoritmo de estimación. Una ventaja adicional en el cálculo de este estimador (al que se llamará índice de Duclos, Esteban y Ray, IDER), dadas sus propiedades asintóticas, es que no depende de la distribución poblacional de la cual se extrae la muestra; en este sentido, su estimación es consistente e independiente de la distribución poblacional subyacente.

Para el caso de las entidades federativas de México, la estimación del IDER se realizó considerando dos escenarios del parámetro $\alpha$ : $\alpha=0$ (correspondiente al coeficiente de Gini) y $\alpha=0.5$, con el objeto de mostrar la correlación entre la polarización y la inequidad simple. Los resultados se consignan en el cuadro 1, junto con los componentes de alienación e identificación, así como la posición relativa que ocupa cada estado en función de su nivel de polarización, lo que permite jerarquizarlos. Adicionalmente, los resultados se muestran en el mapa A1 del anexo.

Se puede observar que existe una variación territorial notable del comportamiento del indicador de polarización. Los estados con mayor grado de polarización son Guerrero, Zacatecas y Campeche, y a la vez se halla un patrón de alta polarización del ingreso en los estados del Sureste, si bien otros fuera de esta región también se encuentran en este caso, como Nuevo León.

Por otro lado los estados de menor polarización son Tlaxcala, Coahuila y Colima. La estimación del IDER, para el parámetro $\alpha=0$ muestra que los casos de mayor inequidad coinciden con los de mayor polarización. Así, Guerrero, 
82 ECONOMÍA: TEORÍA Y PRÁCTICA • Nueva Época, número 45, julio-diciembre 2016

Cuadro 1. Índice de polarización por entidad federativa y sus componentes. México, 2012

\begin{tabular}{|c|c|c|c|c|c|c|c|c|c|}
\hline Entidad & 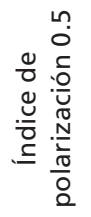 & 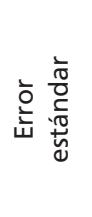 & $\begin{array}{l}: \frac{c}{0} \\
: \underline{u} \\
0 \\
0\end{array}$ & 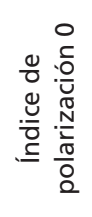 & 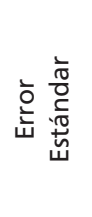 & :0 & 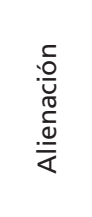 & 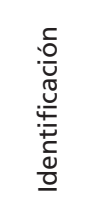 & 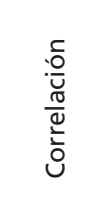 \\
\hline Guerrero & 0.303 & 0.013 & 1 & 0.496 & 0.02 & 2 & 0.496 & 0.601 & 0.017 \\
\hline Zacatecas & 0.295 & 0.007 & 2 & 0.497 & 0.011 & 1 & 0.497 & 0.678 & -0.125 \\
\hline Campeche & 0.291 & 0.012 & 3 & 0.482 & 0.019 & 3 & 0.482 & 0.746 & -0.189 \\
\hline Nuevo León & 0.29 & 0.024 & 4 & 0.461 & 0.027 & 10 & 0.461 & 0.773 & -0.187 \\
\hline Tabasco & 0.289 & 0.011 & 5 & 0.473 & 0.016 & 6 & 0.473 & 0.717 & -0.148 \\
\hline Chiapas & 0.289 & 0.007 & 6 & 0.478 & 0.011 & 5 & 0.478 & 0.584 & 0.034 \\
\hline Oaxaca & 0.288 & 0.009 & 7 & 0.479 & 0.014 & 4 & 0.479 & 0.635 & -0.053 \\
\hline Chihuahua & 0.284 & 0.01 & 8 & 0.469 & 0.016 & 7 & 0.469 & 0.752 & -0.196 \\
\hline Veracruz & 0.283 & 0.015 & 9 & 0.45 & 0.019 & 14 & 0.45 & 0.679 & -0.072 \\
\hline Puebla & 0.282 & 0.013 & 10 & 0.465 & 0.017 & 9 & 0.465 & 0.673 & -0.099 \\
\hline Querétaro & 0.28 & 0.009 & 11 & 0.467 & 0.014 & 8 & 0.467 & 0.767 & -0.218 \\
\hline $\begin{array}{l}\text { Estado de } \\
\text { México }\end{array}$ & 0.279 & 0.025 & 12 & 0.447 & 0.035 & 16 & 0.447 & 0.741 & -0.157 \\
\hline San Luis Potosí & 0.278 & 0.01 & 13 & 0.461 & 0.016 & 11 & 0.461 & 0.699 & -0.136 \\
\hline Hidalgo & 0.272 & 0.01 & 14 & 0.44 & 0.015 & 20 & 0.44 & 0.685 & -0.099 \\
\hline Michoacán & 0.272 & 0.009 & 15 & 0.441 & 0.015 & 18 & 0.441 & 0.688 & -0.106 \\
\hline Aguascalientes & 0.27 & 0.014 & 16 & 0.441 & 0.019 & 17 & 0.441 & 0.737 & -0.169 \\
\hline Durango & 0.27 & 0.008 & 17 & 0.45 & 0.014 & 13 & 0.45 & 0.691 & -0.132 \\
\hline Nayarit & 0.269 & 0.007 & 18 & 0.457 & 0.013 & 12 & 0.457 & 0.693 & -0.15 \\
\hline Jalisco & 0.268 & 0.01 & 19 & 0.441 & 0.013 & 19 & 0.441 & 0.743 & -0.184 \\
\hline Sonora & 0.267 & 0.006 & 20 & 0.449 & 0.011 & 15 & 0.449 & 0.767 & -0.223 \\
\hline Guanajuato & 0.267 & 0.007 & 21 & 0.435 & 0.012 & 22 & 0.435 & 0.73 & -0.16 \\
\hline $\begin{array}{l}\text { Baja California } \\
\text { Sur }\end{array}$ & 0.265 & 0.008 & 22 & 0.438 & 0.015 & 21 & 0.438 & 0.754 & -0.199 \\
\hline Yucatán & 0.264 & 0.007 & 23 & 0.432 & 0.011 & 25 & 0.432 & 0.719 & -0.151 \\
\hline Tamaulipas & 0.263 & 0.007 & 24 & 0.427 & 0.011 & 26 & 0.427 & 0.729 & -0.153 \\
\hline Sinaloa & 0.263 & 0.006 & 25 & 0.432 & 0.01 & 24 & 0.432 & 0.733 & -0.17 \\
\hline Quintana Roo & 0.261 & 0.008 & 26 & 0.434 & 0.015 & 23 & 0.434 & 0.734 & -0.182 \\
\hline Baja California & 0.26 & 0.006 & 27 & 0.427 & 0.011 & 27 & 0.427 & 0.746 & -0.183 \\
\hline Morelos & 0.259 & 0.006 & 28 & 0.411 & 0.011 & 31 & 0.411 & 0.712 & -0.116 \\
\hline $\begin{array}{l}\text { Ciudad de } \\
\text { México }\end{array}$ & 0.259 & 0.007 & 29 & 0.416 & 0.012 & 29 & 0.416 & 0.734 & -0.153 \\
\hline Colima & 0.255 & 0.006 & 30 & 0.419 & 0.01 & 28 & 0.419 & 0.733 & -0.17 \\
\hline Coahuila & 0.254 & 0.008 & 31 & 0.413 & 0.015 & 30 & 0.413 & 0.731 & -0.159 \\
\hline Tlaxcala & 0.251 & 0.007 & 32 & 0.403 & 0.01 & 32 & 0.403 & 0.678 & -0.08 \\
\hline
\end{tabular}

Nota: Los estimados se verificaron estadísticamente con un nivel de significación de $5 \%$. Fuente: Estimación propia con datos del INEGI (2012a). 
Zacatecas y Campeche, además de tener los índices más elevados de polarización, tienen los estimados más elevados de inequidad IDER $\alpha=0$. Sin embargo, en 16 estados, la posición relativa que ocupan los estimados de inequidad difiere de la de polarización. En particular, se pueden distinguir dos grupos: el primero, conformado por estados con un mayor grado de polarización respecto del indicador de inequidad y un segundo grupo en donde esta relación se invierte.

En el primer grupo se encuentran Morelos, Tamaulipas, Yucatán, Hidalgo, Michoacán, Estado de México, Veracruz y Nuevo León, y el segundo grupo está integrado por Oaxaca, Querétaro, San Luis Potosí, Durango, Nayarit, Sonora, Quintana Roo y Colima. En ambos casos, el efecto de alienación tiene una influencia determinante en la posición relativa del índice de polarización; así, entre mayor es el efecto de alienación, mayor resulta el índice de polarización estimado.

\section{Mapa 1. Estados con mayor polarización relativa*}

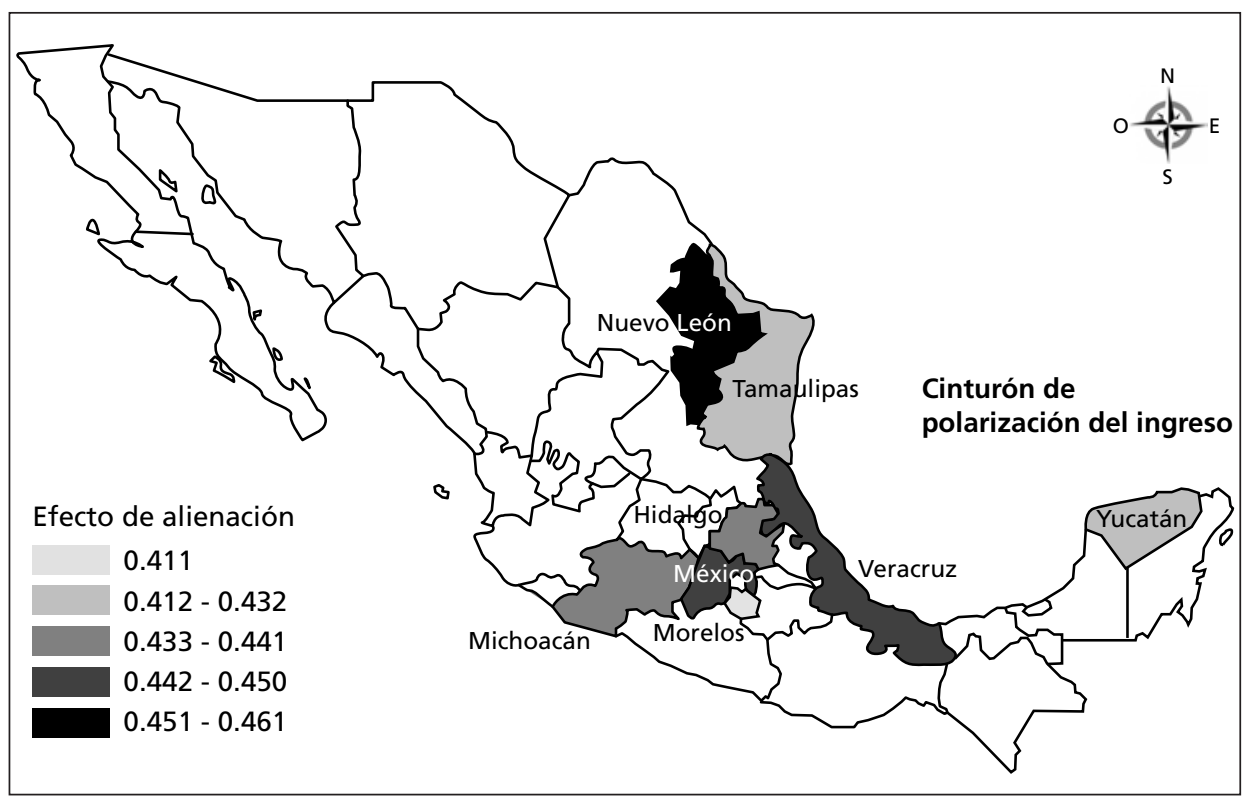

* Polarización relativa se refiere a la posición del IDER ${ }_{\alpha=0.05}$ respecto al IDER ${ }_{\alpha=0}$ dentro de la muestra de 32 entidades.

Nota: El efecto de alienación varía en el intervalo [0,1]; a medida que el valor se aproxima a 1, la distancia entre los niveles de ingreso es mayor.

Fuente: Elaboración propia con datos del INEGI (2012a). 
El mapa 1 muestra los estados que conforman el primer grupo, cuya posición en el ordenamiento de polarización $\operatorname{IDER}_{\alpha}=0.5$ es superior a la que tienen en el ordenamiento para el caso de inequidad simple IDER ${ }_{\alpha=0}$. En él, es posible apreciar que existe un cinturón de alta polarización del ingreso que va desde Michoacán, en el centro occidente del país, hasta Veracruz, pasando por el Estado de México, Morelos e Hidalgo, y que se extiende, hacia el noreste, a Tamaulipas y Nuevo León.

Desde el punto de vista de los efectos que componen el indicador de polarización (alienación e identificación), no parece existir un patrón definido al cual se atribuya la diferencia entre el grupo 1 y el grupo 2. La explicación de esta regularidad empírica encontrada requiere de un análisis más profundo que incorpore otros elementos, tal vez asociados a la estructura del mercado laboral en cada uno de estos estados, por lo que se propone como una línea de investigación futura.

\section{Relación entre el índice de polarización económica y el gasto público federalizado en infraestructura social}

Tal como se evidenció en la sección I, las condiciones de polarización en México difieren significativamente una vez que se analiza el comportamiento de este indicador a escala estatal, por lo que para analizar el impacto que tienen los recursos que a nivel estatal se asignan mediante el gasto público federalizado en los rubros de infraestructura social así como las participaciones que retribuyen la venta de gasolina y diesel, se propone el siguiente modelo, en el que se aplica la técnica de regresión ponderada geográficamente:

$$
\text { ider }_{\alpha 0.05}=\alpha_{0}+\beta_{1} \text { fais }+\beta_{2} \text { faeta }+\beta_{3} \text { fasa }+\beta_{4} \text { pfgas }+\beta_{5} \text { pobreza }+\mu_{1} \text {, }
$$

donde la variable dependiente es el índice de polarización, IDER ${ }_{\alpha 0.05}$, usando el parámetro $\alpha=0.05$. Está normalizado respecto al índice promedio observado por entidad y se expresa como porcentaje.

Las variables independientes fais, faeta y fasa corresponden a los recursos del Fondo de Aportaciones para la Infraestructura Social, al Fondo de Aportaciones para la Educación Tecnológica y de Adultos y al Fondo de Aportaciones para Servicios de Salud, mientras que la variable pfgas consigna los recursos por participaciones de incentivos a la venta final de gasolinas y diesel. Estas variables se expresan en millones de pesos corrientes. La variable pobreza incluye la proporción de población por debajo de la línea de bienestar mínimo considerada 
por el CONEVAL para agosto de 2012 (CONEVAL, 2012) ${ }^{11}$ en su vertiente de canasta alimentaria más la no alimentaria.

En los primeros tres casos, la relación prevista es inversa, mientras que los niveles de pobreza, así como las participaciones de incentivos a la venta de gasolina, se espera que presenten una relación directa con los de polarización, aunque, en este último caso, el efecto redistributivo del fondo de compensación podría atenuar el efecto positivo anticipado. Inicialmente se verifica la especificación del modelo propuesto a partir de la prueba de número condicional, para descartar posibles problemas de multicolinealidad, dada la alta correlación entre las variables independientes fais, faeta y fasa (cuadro 2).

Cuadro 2. Diagnóstico de multicolinealidad del modelo de la ecuación (5)

\begin{tabular}{lcccc}
\hline \multicolumn{1}{c}{ Variable } & $\begin{array}{c}\text { Factor de inflación } \\
\text { de la varianza }\end{array}$ & $\begin{array}{c}\text { Raíz cuadrada del } \\
\text { factor de inflación } \\
\text { de la varianza }\end{array}$ & Tolerancia & $R^{2}$ \\
\hline Fasa & 1.82 & 1.35 & 0.5489 & 0.4511 \\
Faeta & 11.81 & 3.44 & 0.0847 & 0.9153 \\
Fais & 2.15 & 1.46 & 0.4662 & 0.5338 \\
Pobreza & 1.1 & 1.05 & 0.9062 & 0.0938 \\
Ptgas & 6.99 & 2.64 & 0.1432 & 0.8568 \\
\hline Significación del & & & & \\
factor de inflación & 4.77 & & & \\
de la varianza & & & & \\
\hline
\end{tabular}

Fuente. Estimación propia con datos del CONEVAL (2012) y el Presupuesto de Egresos de la Federación (2012).

Se identificó un posible problema de multicolinealidad con la variable faeta a partir del factor de inflación de la varianza (=11.81), por lo que se eligió la siguiente especificación para conducir la regresión ponderada geográficamente:

$$
\operatorname{ider}_{\alpha 0.05}=\alpha_{0}+\beta_{1} \text { fais }+\beta_{2} \text { fasa }+\beta_{3} \text { pfgas }+\beta_{4} \text { pobreza }+\mu_{1}
$$

Los resultados se presentan en los mapas 2 y 3 , y se consignan en el cuadro A3 del anexo. El mapa 2 ilustra la magnitud del coeficiente de la variable fais, mientras que el 3 muestra el impacto de la variable pfgas.

${ }^{11}$ El CONEVAL plantea la línea de bienestar mínimo por tipo de localidad, según sea rural o urbana: en este caso, la estimación se realizó empleando el valor promedio de los dos ámbitos. Asimismo, el cálculo se hizo con base en el valor monetario para agosto de 2012, de MXN 1489.78 , para el ámbito rural, y MXN 2 328.82, para el urbano. 


\section{Mapa 2. Regresión ponderada geográficamente (variable fais)}

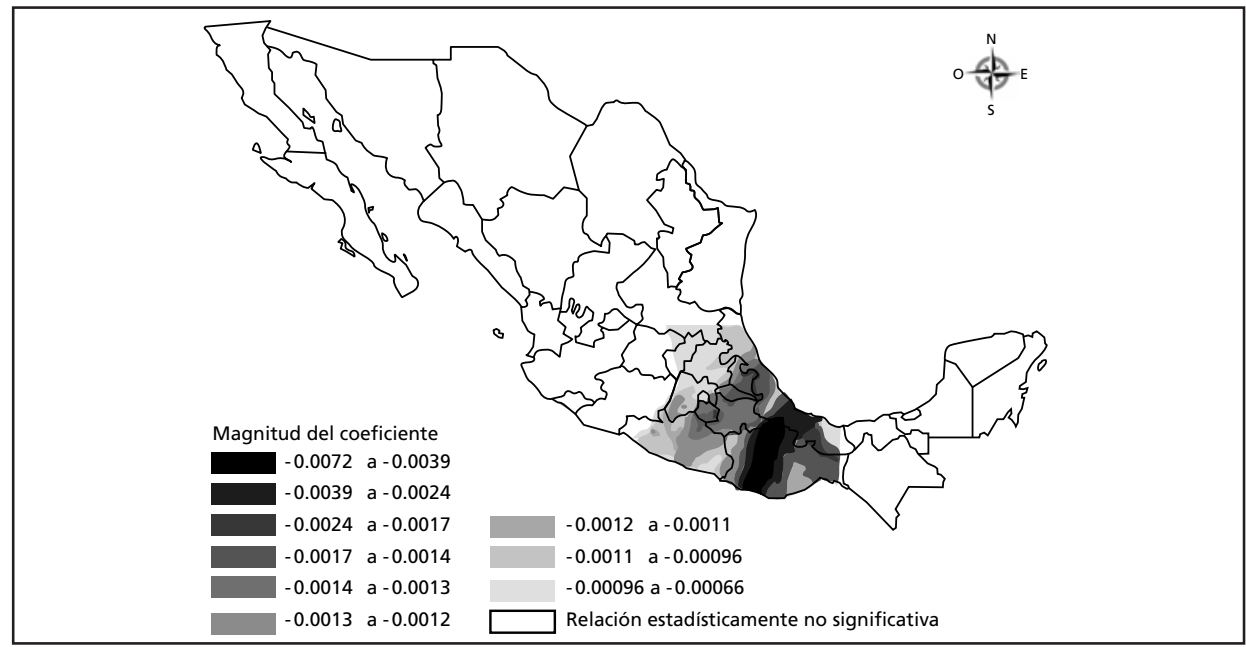

Fuente: Estimación propia con datos del coneval (2012), Presupuesto de Egresos de la Federación (2012) y la ENIGH (INEGI, 2012b).

\section{Mapa 3. Regresión ponderada geográficamente (variable pfgas)}

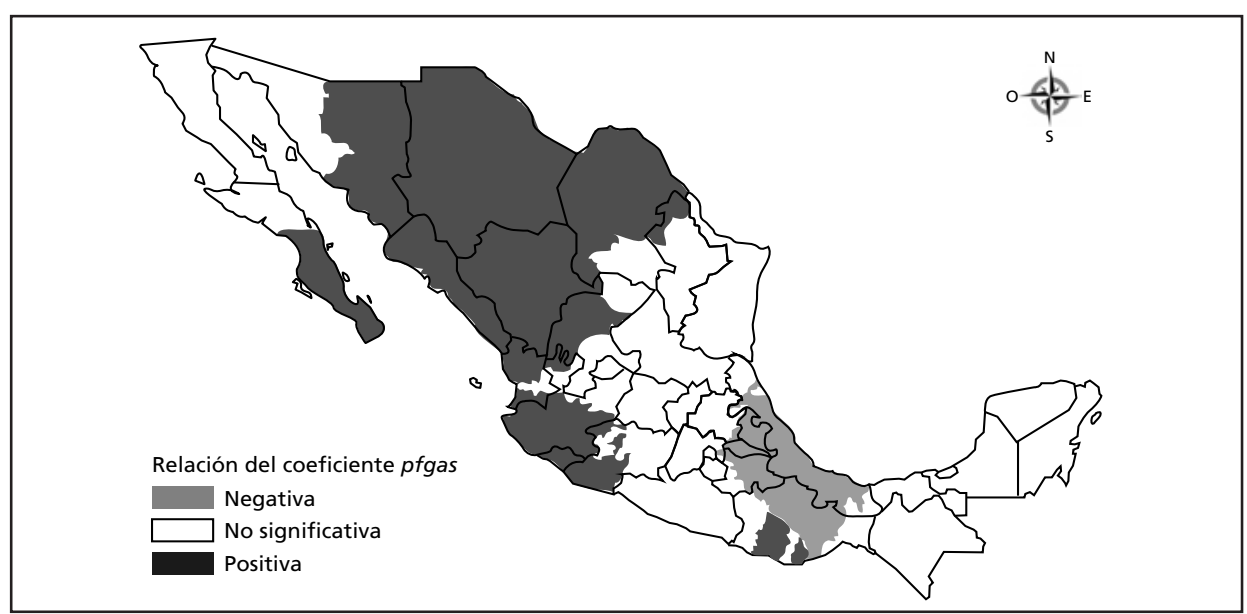

Fuente: Estimación propia con datos del coneval (2012), el Presupuesto de Egresos de la Federación (2012) y la ENIGH (INEGI, 2012b).

Respecto al impacto de los recursos del Fondo de Aportaciones para Infraestructura Social, en el mapa 2 se observan dos elementos destacados: en primer lugar, su efecto pareciera ser limitado a escala nacional, ya que sólo es estadísticamente significativo en un tercio de las entidades federativas: Oaxaca 
Guerrero, Veracruz, Tlaxcala Puebla, Morelos, Estado de México, Hidalgo, Ciudad de México y Querétaro. En segundo lugar, su repercusión se asocia de forma marginal con una reducción de los niveles observados de polarización económica medidos a partir del IDER $\alpha=0.05$ en los estados señalados, en especial en la región del istmo de Tehuantepec.

El mapa 3 muestra la relación entre el coeficiente estimado de la variable participaciones federales de incentivos a la venta final de gasolinas y diesel y el índice de polarización, observándose un comportamiento diferenciado, ya que los coeficientes no resultan estadísticamente significativos en todo el territorio. Así, estos recursos se asocian con mayores índices de polarización en los estados del noroeste, Pacífico y la región de Jalisco, Colima y Michoacán; por el contrario, se asocian con índices menores en Veracruz, Puebla, Tlaxcala y Oaxaca, lo que se pude explicar parcialmente por el efecto del fondo de compensación de este rubro, que se destina a las entidades de menores ingresos. Finalmente, la relación entre las variables fassa, pobreza y el índice de polarización económica es positiva, y resulta estadísticamente significativa para todo el territorio, lo que confirma que en México las condiciones de pobreza están ligadas a escenarios de inequidad extrema.

\section{Estimación de efectos redistributivos del gasto fiscal por tasa negativa de IEPS a gasolina y diesel}

En esta sección se estima el impacto de la redistribución de los recursos implícitos en la tasa negativa del IEPS a gasolina y diesel sobre el ingreso de los hogares. El efecto de esta reasignación se evalúa posteriormente mediante la comparación del índice de polarización del ingreso de 2008 con el de 2012. La estructura de distribución de estos recursos a los hogares se retomó de la distribución observada por el gasto en programas sociales de reconocido carácter progresivo, de acuerdo con los criterios del CONEVAL (2014).

En este sentido, dicha institución ha identificado el nivel de progresividad (o regresividad) que los programas de gasto social tienen en la distribución del ingreso a través de la herramienta denominada Valoración del Desempeño de los Programas de Desarrollo Social (CONeval, 2014). De acuerdo con ese instrumento, los programas de gasto social se clasifican, según su incidencia distributiva, en muy progresivos, progresivos y regresivos. De 137 programas evaluados, sólo siete están valorados como muy progresivos: Programa IMSs-Oportunidades, Seguro Popular, Programa de Abasto Rural a cargo de Diconsa, SA de Cv), Progra- 
ma de Apoyo Alimentario, Programa 70 y Más/Pensión para Adultos Mayores, Programa de Empleo Temporal y Programa de Desarrollo Humano Oportunidades. De ellos, se optó por este último para realizar la estimación empírica, descartando aquellos cuyo universo de aplicación está sesgado a un grupo de beneficiarios reducido, como el caso del Programa de Apoyo Alimentario, que se enfoca en el ámbito rural o el de Empleo Temporal, que sólo opera en un subconjunto de estados en el país.

Cuadro 3. Incidencia redistributiva de recursos por gasto fiscal sobre el índice de polarización, 2008 y 2012

\begin{tabular}{|c|c|c|c|c|c|c|}
\hline Índice & Estimado & $\begin{array}{c}\text { Error } \\
\text { estándar }\end{array}$ & $t$ & $P>t$ & & $\operatorname{Pr}(T<t)$ \\
\hline IDER_Y1 2008 & 0.267 & 0.002 & 161.9 & 0.0 & Ha: estimado (diferencia) $<0$ & 1.0 \\
\hline IDER_Y2 2008 & 0.238 & 0.000 & . & . & Ha: estimado (diferencia) $!=0$ & 0.0 \\
\hline Diferencia & -0.029 & 0.002 & -17.7 & 0.0 & Ha: estimado (diferencia) > 0 & 0.0 \\
\hline IDER_Y1 2012 & 0.265 & 0.003 & 101.7 & 0.0 & Ha: estimado (diferencia) $<0$ & 1.0 \\
\hline IDER_Y2 2012 & 0.236 & 0.000 & . & . & Ha: estimado (diferencia) $!=0$ & 0.0 \\
\hline Diferencia & -0.029 & 0.003 & -11.2 & 0.0 & Ha: estimado (diferencia) $>0$ & 0.0 \\
\hline
\end{tabular}

Nota: IDER_Y1; Índice de polarización para ingreso por hogar sin transferencia por IEPS. IDER_Y2: Índice de polarización para ingreso por hogar con transferencia por IEPS.

Fuente: Estimación propia con datos del Módulo de Condiciones Socioeconómicas (INEGI, 2008; 2012a), el Presupuesto de Gastos Fiscales (SHCP, 2008; 2012a) y datos de 2008 y 2009 del CONEVAL (2014).

Se encuentra que durante el periodo 2008-2012, que abarca una fase recesiva del ciclo económico, las condiciones de polarización no han mejorado y permanecieron alrededor de IDER $\approx 0.26$. No obstante, se observa un impacto estadísticamente significativo en la reducción de la polarización, atribuible a la reasignación de los recursos por la tasa negativa de IEPS, en ambos años, al pasar de IDER $\approx 0.26$ a IDER $\approx 0.23$ en dicho lapso.

Una ventaja adicional de esta selección es que a través del Módulo de Condiciones Socioeconómicas es posible conocer la asignación de recursos a los hogares por este programa, lo que permite contar con la estructura distributiva base para asignar los recursos del IEPS conforme al mismo patrón. En primer lugar, se estimó el índice de polarización para el país entre 2008 y 2012 con el objeto de verificar el comportamiento de esta variable en el tiempo y, en segundo, se compararon los niveles de polarización una vez que se aplicaron los recursos del IEPS al ingreso. Los resultados se presentan en el cuadro 3. La columna co- 
rrespondiente al IDER_y1 muestra el nivel de polarización antes de la reasignación de los recursos por el IEPS, mientras que la columna IDER_y2 muestra el nivel de polarización una vez reasignados.

\section{DiSCUSIÓN DE RESULTADOS Y CONCLUSIONES}

El presente trabajo se inserta en las propuestas de mejorar la distribución del ingreso en México como mecanismo para capitalizar los beneficios del crecimiento y maximizar el impacto de las políticas de gasto publico orientadas a combatir los rezagos sociales y la pobreza. Retoma los esfuerzos realizados, para el caso de México, por autores como Huesca (2005) y Carrillo-Huerta y Vázquez (2005), y amplía el análisis para incluir la perspectiva territorial, como lo sugieren recientemente Fuentes y Fuentes (2010).

El trabajo se apoya en dos pilares conceptuales: el tema de gastos fiscales y la caracterización territorial de la polarización del ingreso en México. En particular, el análisis del caso de la tasa negativa del IEPS a gasolina y diesel permite inferir el potencial redistributivo de este gasto fiscal y la necesidad de rediseñar su aplicación.

Por otra parte, se encuentra que las condiciones de extrema inequidad económica, medidas con el IDER, tienen un comportamiento diferenciado, aunque el análisis de los componentes de este índice permitió identificar un conjunto de estados en los que existen condiciones de polarización más acentuadas y que integran un cinturón que se extiende desde Michoacán hasta Veracruz y Tamaulipas. Respecto a los esfuerzos redistributivos que el gobierno federal realiza mediante el Ramo 28 (participaciones federales) y el Ramo 33 (aportaciones federales) del Presupuesto de Egresos, se encontró que el FaIs tiene un impacto limitado a escala nacional, ya que sólo es estadísticamente significativo en un tercio de las entidades federativas en relación con los niveles de polarización económica. Sin embargo, en las que sí tiene una relación significativa, el impacto redistributivo es el esperado, con una relación inversa respecto a los niveles observados de polarización, en especial, en la región del istmo de Tehuantepec.

Por su parte, la relación entre las participaciones federales por incentivos a la venta final de gasolinas y diesel y el índice de polarización permite apreciar la influencia del fondo de compensación que se destina a las entidades de menores ingresos, ya que se encontró una relación inversa con los índices de polarización en los estados de Veracruz, Puebla, Tlaxcala y Oaxaca. No obstante, predomina la influencia del criterio de retribución al consumo, ya que esta varia- 
ble se asocia con mayores índices de polarización en los estados del noroeste, del Pacífico y la región de Jalisco, Colima y Michoacán. Adicionalmente, se encuentra que los niveles de pobreza y el índice de polarización tienen una vinculación directa; las estimaciones demuestran que la relación es estadísticamente significativa en todas las entidades, lo que confirma que las condiciones de pobreza están ligadas a los escenarios de inequidad.

Finalmente, el análisis de los efectos redistributivos del gasto fiscal que implica la tasa negativa del IEPS a gasolina y diesel durante la fase recesiva de 2008 a 2012 permitió corroborar un impacto estadísticamente significativo en la reducción de la polarización para ambos años al pasar de IDER $\approx 0.26$ a IDER $\approx 0.23$ en dicho periodo. Estos hallazgos permiten subrayar la importancia del diseño regresivo de los tratamientos fiscales diferenciales. En síntesis, el planteamiento expuesto enfatiza la necesidad de implementar mecanismos redistributivos que permitan maximizar los beneficios del crecimiento económico y, en particular, el impacto de los recursos asignados mediante políticas redistributivas en favor de los pobres, hacia las que México aparentemente se ha orientado en tiempos recientes.

\section{ANEXo}

\section{Mapa A1. Índice de polarización por entidad federativa}

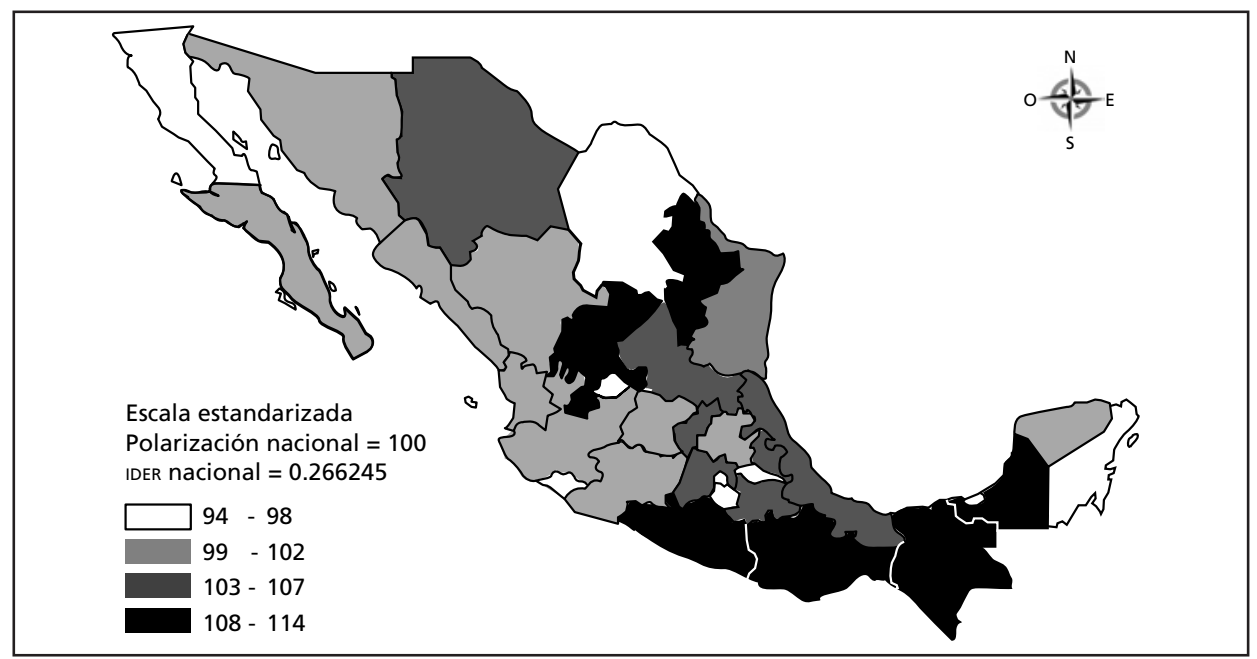

Nota: Resultados para el IDER considerando el parámetro $\alpha=0.5$.

Fuente: Elaboración propia con base en información del INEGI (2012a; 2012b). 
Cuadro A1. Publicaciones que estiman la polarización del ingreso en México

\begin{tabular}{l|l}
\hline \multicolumn{1}{c|}{ Autor } & \multicolumn{1}{c}{ Metodología para estimar la polarización } \\
\hline Huesca (2003) & $\begin{array}{l}\text { Índice de Esteban y Ray; índice de Esteban, } \\
\text { Gradín y Ray; estimación paramétrica. }\end{array}$ \\
\hline Huesca (2005) & $\begin{array}{l}\text { Índice de Esteban y Ray, estimación } \\
\text { paramétrica. }\end{array}$ \\
\hline Carrillo-Huerta y Vázquez (2005) & $\begin{array}{l}\text { Índice de Esteban y Ray; índice de Gradín } \\
\text { y Rossi; índice de Esteban, Gradín y Ray; } \\
\text { estimación paramétrica. }\end{array}$ \\
\hline Fuentes y Fuentes (2010) & $\begin{array}{l}\text { Índice de Esteban y Ray, estimación } \\
\text { paramétrica. }\end{array}$ \\
\hline
\end{tabular}

Cuadro A2. Prueba de diferencia de medias para componentes del índice de polarización e ingreso mensual medio por hogar

\begin{tabular}{lcccc}
\hline \multicolumn{1}{c}{ Grupo } & Observaciones & $\begin{array}{c}\text { Alienación } \\
\text { media }\end{array}$ & $\begin{array}{c}\text { Identificación } \\
\text { media }\end{array}$ & Ingreso \\
\hline IDER $0.5>$ IDER 0 & 8 & 0.439 & 0.716 & MXN 14113.90 \\
IDER $0>$ IDER 0.5 & 8 & 0.452 & 0.715 & MXN 14345.40 \\
Combinado & 16 & 0.445 & 0.715 & MXN 14229.65 \\
Diferencia & & -0.013 & 0.001 & -231.5086 \\
Ho: diferencia $=0$ & $t$ & -1.531 & 0.050 & -0.133 \\
Ha: diferencia !=0 & & 0.140 & 0.960 & 0.8961 \\
$\operatorname{Pr}(|T|>|t|)$ & & &
\end{tabular}

Fuente: Estimación propia con datos de la ENIGH (INEGI, 2012b).

Cuadro A3. Modelo de regresión ponderada geográficamente

\begin{tabular}{lcll}
\hline \multicolumn{1}{c}{ Variable } & Parámetros & Dependiente & IDER 0.05 \\
\hline Suma de los cuadrados de los residuos & 307.2962 & Explicativa 1 & fais \\
Número efectivo & 21.0962 & Explicativa 2 & fassa \\
$\sigma$ & 0.3553 & Explicativa 3 & pobreza \\
$R^{2}$ & 0.5264 & Explicativa 4 & pfgas \\
$R^{2}$ ajustada & 0.5225 & & \\
\hline
\end{tabular}

Fuente: Estimación propia con datos del coneval (2012), el Presupuesto de Egresos de la Federación (2012) y la ENIGH (INEGI, 2012b). 
92 ECONOMÍA: TEORÍA Y PRÁCTICA • Nueva Época, número 45, julio-diciembre 2016

Cuadro A4. Resultados de la regresión ponderada geográficamente

\begin{tabular}{|c|c|c|c|c|c|c|c|c|c|}
\hline Entidad & $r 2$ & c1_fais & c2_fassa & c3_pob & c4_pfgas & $\begin{array}{c}\text { Error } \\
\text { estándar } \\
\text { c1 }\end{array}$ & $\begin{array}{c}\text { Error } \\
\text { estándar } \\
\text { c2 }\end{array}$ & $\begin{array}{c}\text { Error } \\
\text { estándar } \\
\text { c3 }\end{array}$ & $\begin{array}{c}\text { Error } \\
\text { estándar } \\
\text { c4 }\end{array}$ \\
\hline Aguascalientes & 0.49 & -0.000054 & $0.001103 *$ & $2.146^{*}$ & 0.001642 & 0.0005 & 0.0004 & 0.0705 & 0.0009 \\
\hline Baja California & 0.60 & -0.000212 & 0.000572 & $3.09507 *$ & 0.001321 & 0.0018 & 0.0011 & 0.1981 & 0.0018 \\
\hline \multicolumn{10}{|l|}{ Baja California } \\
\hline Sur & 0.58 & -0.000436 & 0.000915 & $2.46148 *$ & $0.001936 *$ & 0.0007 & 0.0005 & 0.0997 & 0.0009 \\
\hline Campeche & 0.51 & -0.000293 & 0.002045 & $1.7944^{*}$ & 0.000907 & 0.0004 & 0.0014 & 0.0697 & 0.0045 \\
\hline $\begin{array}{l}\text { Ciudad } \\
\text { de México }\end{array}$ & 0.49 & $-0.00115^{*}$ & $0.002585^{*}$ & $1.96218^{*}$ & 0.000102 & 0.0005 & 0.0010 & 0.0660 & 0.0024 \\
\hline Colima & 0.50 & $-0.000694 *$ & $0.001068 *$ & $2.06179 *$ & $0.002203 *$ & 0.0003 & 0.0004 & 0.0657 & 0.0010 \\
\hline Chiapas & 0.51 & -0.000225 & $0.003019 *$ & $1.66197 *$ & -0.000543 & 0.0004 & 0.0015 & 0.0667 & 0.0045 \\
\hline Chihuahua & 0.55 & -0.000348 & 0.000625 & $2.40769 *$ & $0.002754 *$ & 0.0006 & 0.0005 & 0.0933 & 0.0009 \\
\hline Durango & 0.53 & -0.000175 & 0.000837 & $2.2543^{*}$ & $0.002285^{*}$ & 0.0006 & 0.0004 & 0.0760 & 0.0009 \\
\hline $\begin{array}{l}\text { Estado } \\
\text { de México }\end{array}$ & 0.49 & $-0.00113^{*}$ & $0.003395 *$ & $1.9643 *$ & -0.002082 & 0.0004 & 0.0010 & 0.0659 & 0.0025 \\
\hline Guanajuato & 0.49 & -0.000539 & $0.001524 *$ & 2.08117 * & 0.001114 & 0.0004 & 0.0005 & 0.0669 & 0.0012 \\
\hline Guerrero & 0.47 & $-0.0012^{*}$ & $0.002767^{*}$ & $1.87479 *$ & -0.000238 & 0.0005 & 0.0010 & 0.0663 & 0.0023 \\
\hline Hidalgo & 0.49 & $-0.001043 *$ & $0.004058^{*}$ & $1.9904^{*}$ & -0.003976 & 0.0004 & 0.0011 & 0.0669 & 0.0027 \\
\hline Jalisco & 0.50 & -0.000608 & $0.001109 *$ & $2.09561 *$ & $0.001993 *$ & 0.0004 & 0.0004 & 0.0671 & 0.0010 \\
\hline Michoa & 0.49 & $-0.000784^{*}$ & $0.001352 *$ & $2.0209 *$ & 0.001814 & 0.0003 & 0.0005 & 0.0653 & 0.0011 \\
\hline Morelos & 0.50 & $-0.001398 *$ & $0.003086^{*}$ & 1.92124 * & -0.000918 & 0.0005 & 0.0010 & 0.0655 & 0.0024 \\
\hline Nayarit & 0.52 & -0.000345 & $0.001017^{*}$ & $2.17185^{*}$ & 0.001951 * & 0.0005 & 0.0004 & 0.0715 & 0.0009 \\
\hline Nuevo León & 0.49 & -0.000094 & $0.001212^{*}$ & $2.13527^{*}$ & 0.001392 & 0.0006 & 0.0005 & 0.0726 & 0.0012 \\
\hline Oaxaca & 0.50 & $-0.00325^{*}$ & $0.009932 *$ & $1.73643^{*}$ & -0.009687 & 0.0009 & 0.0022 & 0.0660 & 0.0054 \\
\hline Puebla & 0.49 & $-0.001519 *$ & $0.006158 *$ & 1.92837 * & $-0.008562 *$ & 0.0005 & 0.0013 & 0.0687 & 0.0032 \\
\hline Querétaro & 9 & -0.000648 & $0.002197^{*}$ & $2.06627^{*}$ & -0.0002 & 0.0004 & 0.0008 & 0.0671 & 0.0019 \\
\hline Quintana Roo & 0.51 & -0.0005 & 0.001836 & $1.87735^{*}$ & 0.001044 & 0.0004 & 0.0015 & 0.0782 & 0.0046 \\
\hline San Luis Potosí & 0.48 & -0.000363 & $0.001709 *$ & $2.09472 *$ & 0.000611 & 0.0004 & 0.0006 & 0.0691 & 0.0015 \\
\hline Sinaloa & 0.55 & -0.000367 & 0.000838 & $2.33354^{*}$ & $0.002354 *$ & 0.0006 & 0.0004 & 0.0824 & 0.0009 \\
\hline Sonora & 0.58 & -0.000284 & 0.000656 & $2.58719 *$ & 0.002201 * & 0.0008 & 0.0005 & 0.1194 & 0.0010 \\
\hline Tabasco & 0.51 & -0.000224 & 0.00269 & $1.71336^{*}$ & -0.000027 & 0.0004 & 0.0014 & 0.0670 & 0.0044 \\
\hline Tamaulipas & 0.47 & -0.000493 & $0.001688^{*}$ & $2.09773 *$ & 0.000799 & 0.0004 & 0.0006 & 0.0702 & 0.0015 \\
\hline Tlaxcala & 0.50 & $-0.001452 *$ & $0.005776 *$ & $1.94727 *$ & $-0.007816^{*}$ & 0.0005 & 0.0012 & 0.0683 & 0.0031 \\
\hline Veracruz & 0.50 & $-0.001622^{*}$ & $0.005305^{*}$ & $1.93458 *$ & -0.004526 & 0.0006 & 0.0013 & 0.0705 & 0.0032 \\
\hline Yucatán & 0.51 & -0.00042 & 0.001795 & $1.85598 *$ & 0.001388 & 0.0004 & 0.0014 & 0.0727 & 0.0044 \\
\hline Zacatecas & 0.51 & -0.000011 & $0.001017^{*}$ & 2.17446 * & 0.001775 & 0.0005 & 0.0004 & 0.0718 & 0.0009 \\
\hline
\end{tabular}

* Coeficiente estadísticamente significativo a 0.05 .

Fuente: Estimación propia con datos del CONEVAL (2012), el Presupuesto de Egresos de la Federación (2012) y la ENIGH (INEGI, 2012b). 


\section{REFERENCIAS BIBLIOGRÁFICAS}

Agostini, Claudio Andrés, y Jiménez, Johanna (2015), “The Distributional Incidence of the Gasoline Tax in Chile", Energy Policy, 85, pp. 243-252.

Antón-Sarabia, Arturo, y Hernández-Trillo, Fausto (2014), "Optimal Gasoline Tax in Developing, Oil-producing Countries: The Case of Mexico", Energy Policy, 67, pp. 564-571.

Atkinson, Anthony Barnes (1997), "Bringing Income Distribution in from the Cold", The Economic Journal, 107 (441), pp. 297-321.

Banxico (s.f.), "Tipo de cambio para solventar obligaciones denominadas en dólares de los EeUuA, pagaderas en la República Mexicana". Consultado el 15 de febrero de 2014, en: http://www.banxico.org.mx/tipcamb/tipCamIHAction.do.

Bourguignon, François, y Morrison, Christian (2002), "Inequality Among World Citizens: 1820-1992", American Economic Review, 92 (4), pp. 727-44.

Carrillo-Huerta, Mario, y Vázquez, Haydee (2005), "Desigualdad y polarización en la distribución del ingreso salarial en México", Problemas del Desarrollo, 36 (141), pp. 109-130.

Clementi, Fabio, y Schettino, Francesco (2013), "Income Polarization in Brazil, 2001-2011: A Distributional Analysis Using PNAD Data”, Economics Bulletin, 33 (3), pp. 1796-1815.

CONEval (2012), "Evolución de las líneas de bienestar y de la canasta alimentaria". Consultado el 25 de febrero de 2014 en: http://www.coneval.gob.mx/Medicion/ Paginas/Lineas-de-bienestar-y-canasta-basica.aspx.

(2014), "Inventario CONEval de programas y acciones federales de desarrollo social". Consultado el 25 de febrero de 2014 en: http://www.coneval.gob.mx/ evaluacion/ipfe/Paginas/default.aspx.

Duclos, Jean-Yves, Joan, Esteban, y Ray, Debraj (2004), "Polarization: Concepts, Measurement, Estimation”, Econometrica, 72 (6), pp. 1737-1772.

Esteban, Joan-Maria; Gradín, Carlos, y Ray, Debraj (1999), "Extensions of a Measure of Polarization, with an Application to the Income Distribution of Five OECD Countries", Luxembourg Income Study Working Paper Series 218, Maxwell School of Citizenship and Public Affairs, Syracuse University.

Esteban, Joan-Maria, y Ray, Debraj (1994), "On the Measurement of Polarization", Econometrica, 62 (4), pp. 819-851.

Fuentes, Noé Arón, y Fuentes, Melina (2010), “Polarización económica regional en México: 1980-2004”, Cofactor, 1 (2), pp. 7-22. 
Gasparini, Leonardo; Cruces, Guillermo; Tornarolli, Leopoldo, y Marchionni, Mariana (2009), “A Turning Point? Recent Developments on Inequality in Latin America and the Caribbean", CEDLAs Working Papers 81, CEDLAS.

Huesca, Luis (2003), “Análisis de la polarización del ingreso de los hogares en México durante 1984-2000”, Investigación Económica, 62 (246), pp. 89-123.

(2005), “Polarización por subgrupos socioeconómicos en México: 1984-2002”, Estudios Sociales, 13 (25), pp. 36-68.

IMF (2007), Manual on Fiscal Transparency, Washington, DC, International Monetary Fund Publication Services. [en línea] Consultado el 25 de febrero de 2014, en: http://www.imf.org/external/np/pp/2007/eng/101907m.pdf.

INEGI (2008), "Módulo de Condiciones Socioeconómicas (MCs) 2008”, en Encuestas en hogares. Consultado el 21 de febrero de 2014, en: http://www.inegi.org.mx/est/ contenidos/proyectos/encuestas/hogares/modulos/mcs/mcs2008/default.aspx. (2012a), "México - Módulo de Condiciones Socioeconómicas de la ENIGH", en Red Nacional de Metadatos. Consultado el 21 de febrero de 2014, en: http:// www3.inegi.org.mx/rnm/index.php/catalog/76.

(2012b), "Encuesta Nacional de Ingresos y Gastos de los Hogares (ENIGH)". Consultado el 26 de febrero de 2014, en: http://www.inegi.org.mx/est/ contenidos/Proyectos/encuestas/hogares/regulares/enigh/.

Kakwani, Nanak (1976), "Measurement of Tax Progressivity: An International Comparison”, The Economic Journal, 87 (345), pp. 71-80.

Kraan, Dirk-Jan (2004), “Off-budget and Tax Expenditures”, oECD Journal of Budgeting, 4 (1), pp. 121-142.

Ley de Coordinación Fiscal (1978). [en línea] Consultado el 16 de octubre de 2013, en http://www.sat.gob.mx/informacion_fiscal/normatividad/Documents/LCF.pdf .

Ley de Ingresos de la Federación para el Ejercicio Fiscal de 2002 (2002), Diario Oficial de la Federación, México, 1 de enero. [en línea] Consultado el 16 de noviembre de 2013, en: http://www.transparenciapresupuestaria.gob.mx/work/models/PTP/ Presupuesto/LIF/Lif_2002.pdf.

Ley de Ingresos de la Federación para el Ejercicio Fiscal de 2013 (2012), Diario Oficial de la Federación, México, 17 de diciembre. [en línea] Consultado el 10 de octubre de 2013, en: http://www.shcp.gob.mx/INGRESOS/Ingresos_ley/2013/ lif_2013.pdf.

Litschig, Stephan, y Morrison, Kevin M. (2013), "The Impact of Intergovernmental Transfers on Education Outcomes and Poverty Reduction", American Economic Journal: Applied Economics, 5 (4), pp. 206-240.

López-Calva, Luis, y Rocha, Sonia (2012), "Exiting BELINDIA? Lesson from the Recent 
Decline in Income Inequality in Brazil", en El Banco Mundial. Documentos e informes. Consultado el 13 de enero de 2014, en: http://documentos. bancomundial.org/curated/es/2012/01/16423641/exiting-belindia-lessonrecent-decline-income-inequality-brazil.

Mijangos, María (1991), "Los gastos fiscales. Concepto y aplicación en México", Boletín Mexicano de Derecho Comparado, 0 (71). [en línea] Consultado el 6 de marzo de 2014, en: http://biblio.juridicas.unam.mx/revista/pdf/derechocomparado/71/ art/art3.pdf.

Musgrave, Richard Abel (1969), Fiscal Systems, New Haven, Yale University Press. (1990), "Horizontal Equity Once More", National Tax Journal, 43 (2), pp. 113-122. OECD (2015), "The Case for Measuring Support for Fossil Fuels", en oECD Companion to the Inventory of Support Measures for Fossil Fuels 2015. [en línea] Consultado el 16 de octubre de 2015, en: http://www.oecd-ilibrary.org/energy/ oecd-companion-to-the-inventory-of-support-measures-for-fossil-fuels-2015/ the-case-for-measuring-support-for-fossil-fuels_9789264239616-4-en.

Organización Mundial de la Salud (2008), Derechos humanos, salud y estrategias de reducción de la pobreza, Serie de Publicaciones sobre Salud y Derechos Humanos 5, Francia, Organización Mundial de la Salud.

Parry, Ian, y Small, Kenneth (2005), "Does Britain or the United States Have the Right Gasoline Tax?", American Economic Review, 95 (4), pp. 1276-1289.

Presupuesto de Egresos de la Federación (2012), Diario Oficial de la Federación, México, 12 de diciembre.

Ravallion, Martin; Thorbecke, Erik, y Pritchett, Lant (2004), Competing Concepts of Inequality in the Globalization Debate, s.l., Brookings Institution Press.

Secretaría de Energía (2012), Prospectiva de petrolíferos 2012-2026, México, DF, SENER. Sen, Amartya (1973), On Economic Inequality, Oxford, Clarendon Press. SHCP (2008), Presupuesto de Gastos Fiscales 2008. [en línea] Consultado el 3 de octubre de 2014, en: http://www.shcp.gob.mx/INGRESOS/Ingresos_pres_gasto/ presupuesto_gastos_fiscales_2008.pdf.

(2009), Presupuesto de Gastos Fiscales 2009. [en línea]. Consultado el 3 de octubre de 2014, en: http://www.shcp.gob.mx/INGRESOS/Ingresos_pres_ gasto/presupuesto_gastos_fiscales_2009.pdf.

(2010), Presupuesto de Gastos Fiscales 2010. [en línea] Consultado el 3 de octubre de 2014, en: http://www.shcp.gob.mx/INGRESOS/Ingresos_pres_ gasto/presupuesto_gasto_fiscales_2010.pdf.

(2011), Presupuesto de Gastos Fiscales 2011. [en línea] Consultado el 3 de 
octubre de 2014, en: http://www.shcp.gob.mx/INGRESOS/ingresos_presupuesto gastos/presupuesto_gastos_fiscales_2011.pdf.

(2012a), Presupuesto de Gastos Fiscales 2012. [en línea] Consultado el 3 de octubre de 2014, en: http://www.shcp.gob.mx/INGRESOS/Ingresos_pres_gasto/ pres_gast_fis_2012.pdf.

(2012b), "El sábado 8 de diciembre los precios de las gasolinas y el diesel aumentarán 9 centavos, el mismo monto que el mes anterior", comunicado de prensa 079/2012, 7 de diciembre. [en línea] Consultado el 10 de diciembre en: http://www.hacienda.gob.mx/SALAPRENSA/doc_comunicados_prensa/2012/ diciembre/comunicado_079_2012.pdf.

Surrey, Stanley (1973), Pathways to Tax Reform: The Concept of Tax Expenditures, Cambridge, Harvard University Press.

Tépach, Reyes (2013), El Presupuesto federalizado para los estados del país y el Distrito Federal, 2012-2013, México, DF, Cámara de Diputados. [en línea] Consultado el 3 de octubre de 2014, en: http://www.diputados.gob.mx/sedia/sia/se/SAEISS-04-13.pdf.

The World Bank (s.f.), “GDP Growth (annual \%)", en Data. Consultado el 15 de enero de 2014, en: http://data.worldbank.org/indicator/NY.GDP.MKTP.KD.ZG?end=201 2\&locations $=$ MX\&start $=2000$.

us Energy Information Administration (s.f.), "Data", en Petroleum \& Other Liquids. Consultado el 10 de enero de 2014, en: https://www.eia.gov/dnav/pet/pet_pri_ spt_sl_m.htm. 